\title{
Microfluidics, microscopy and modeling
}

Researchers use microfluidics, a fluorescent reporter and modeling to quantify yeast's response to glucose availability.

Gene-regulatory networks shoulder much of the responsibility for the adaptability of species, but these complex systems are very difficult to unravel. But by applying new technologies and computational modeling to even well-studied networks, researchers can learn new things.

Jeff Hasty and his colleagues at the University of California, San Diego used a microfluidics platform in combination with fluorescence microscopy to look at the response of the galactose-utilization network in Saccharomyces cerevisiae to sinusoidal changes in glucose concentration over a steady galactose background. The control offered by microfluidics allowed them to generate either slow or fast changes in glucose concentration, over periods ranging from 0.75 to 4.5 hours. They fused the native Gall enzyme to a fluorescent reporter to monitor how the galactose network responded.
The researchers observed that the yeast responded to slow oscillations in glucose concentration by either increasing or decreasing GAL1 transcription, but the yeast did not respond to fast changes. "There's an energy cost for firing up the galactose network," explains Hasty. "Maybe there's some frequency at which it's more energy efficient for the cells to just ignore it."

They put this information into a model of the galactose network, which predicted that the degradation of some network components should be upregulated as glucose concentration increased. They experimentally tested the prediction by monitoring mRNA degradation rates for GAL1 and GAL3 and found that the model was right. "A lot of times modeling can lead you to these [hypotheses], whereas just trying to think your way through these complicated situations won't," says Hasty.

The researchers also used the model to predict how changes in galactose uptake capabilities might affect the network, which could lead to insights into the mechanisms of adaptability. Notably, they found that a yeast strain deficient in Gal2, with impaired galactose uptake capacity, had essentially the same amplitude response to changes in glucose concentration as the normal strain.

Hasty notes that such a combination of microfluidics and fluorescent reporters can be used to study any system that responds to an inducer and has a fluorescent readout. However, without standardized microfluidics technology, applications have been largely limited to the experts. "But increasingly, there are microfluidics people in every department," notes Hasty. "So I think as biologists begin to see how microfluidics can be used, it's going to take off like crazy."

\section{Allison Doerr}

\section{RESEARCH PAPERS}

Bennett, M.R. et al. Metabolic gene regulation in a dynamically changing environment. Nature 454 , 1119-1122 (2008). 